\title{
An Efficient Lossless Compression algorithm for Medical images
}

\author{
Amanpreet Kaur Sandhu \\ Assistant Professor, University Institute of Computing, Chandigarh University, Mohali, Punjab
}

Article History: Received: 10 November 2020; Revised 12 January 2021 Accepted: 27 January 2021; Published online: 5 April 2021

\begin{abstract}
Medical image compression plays a vital role in diagnosis of diseases which allowing manipulation, efficient, transmission and storage of color, binary and grayscale image. Before transmission and storage, a medical image may be required to be compressed. The objective of the study is to develop an efficient and effective technique for digital medical images which alleviates the blocking artifacts from grayscale image while retaining all relevant structures. In this paper, we demonstrate a highly engineered postprocessing filtering approach has been designed to remove blocking effects from medical images at low bit rate. The proposed technique is comprised of three strategies i.e. 1) a threshold valve scheme which is used to capture the pixel vectors containing blocking artifacts. 2) Blocking artifacts measurement techniques. The blocking artifacts are measured by three frequency related modes (low, Moderate and high frequency model). 3) A directional filter which is used to remove over-smoothing and ringing artifacts near edges of block boundary. The algorithm is tested on digital medical grayscale images from different modalities. The experimental results illustrate that the proposed technique is more efficient on the basis of PSNR-B, MSSIM, and MOS indices than the state-of-the-art methods. The proposed algorithm can be seamlessly applied in area of medical image compression which high transmission efficiency and acceptable image quality can be guaranteed.
\end{abstract}

Keywords: Image compression, Magnetic resonance imagining, X-ray and tomography

\section{Introduction}

Image compression is a technique which is used to reduce redundancies in images and represent it in shorter manner, which can allow more cost-effective for storage capacity and utilization of network bandwidth. Therefore, medical image compression plays an important role in many applications [1]. Telemedicine and medical imaging are developing day by day on a very large scale. Teleradiology is mostly used in telemedicine due to its clinical aspects. The aim of Teleradiology is to transfer the medical images like MRI, X-day by day on a very large scale.

Teleradiology is mostly used in telemedicine due to its clinical aspects. The aim of Teleradiology is to transfer the medical images like MRI, X-rays, CT scans and US etc. from one place to another place. These transfers can do from one clinic to another clinic, from an imaging center to the clinic, or from clinic to an imaging center. Thus, it is very crucial to compress these hugh volume of medical image data for storage and transmission. On the other hand, these medical datasets may not be afford to lose any visually significant information which otherwise would lead to wrong diagnosis of any critical pathological condition. Hence, it is very important to maintain the balance between compression efficiency and quality of reconstructed image data [2]. For 16-bit CT image, 256 MB is required for a $512 \times 512$. Mostly, medical images are saved on servers and send to client when required [3]. The objective of the medical image compression algorithms is to reduce the huge number of datasets to be stored, transferred and processed while preserving diagnostic information. The image compression algorithm can be categorized into two parts: lossless and lossy. Lossless algorithm is associated with reversible and noiseless coding which provides complete recovery of the original datasets from compressed datasets. It may be used in those cases where the data size is not heavy. Lossy algorithms generate some errors or crash the datasets during the compression process. These methods are suitable for large data sets with high compression ratios.

\section{Previous Work}

Various advance image compression techniques have been designed in response to the increasing demand for medical images. Jiang et al. [4] designed multiple transmission optimization scheme for medical images called MTO which analysis the visual content of multiple images based on the characteristics of recourse constraint mobile telemedicine system and medical image. Yadav et al. [5] designed a hybrid technique for image compression for medical images to increase compression ratio with the easy computational burden with good decoded quality. Lucas et al. [6] described a lossless compression of volumetric sets of medical images using 3D minimum rate predictors. Placidi [7] proposed a lossless compression algorithm from projections which eliminates 
both redundant information and noise for medical images. Sumalatha et al. [8] used an adaptive multiwavelet transform (AMWT) with multidimensional layered zero coding (MLZC). Singh et al. proposed a technique on the basis of DCT blocks which is classified into two parts pure and complex regions. While pure regions utilize numerous of images with small information content and complex regions used many coefficients to maintain the diagnostic information [9]. Lim et al. [10] constructed a principal component analysis (PCA) algorithm which is applied on each block of an image. To reduce the computational complexity of DCT based compression methods that subdivide an image into non-overlapping blocks of fixed size for example $8 \times 8,16 \times 16$ pixels. The DCT processed all blocks and quantization implemented to transform coefficients [11-15]. The drawback of these techniques is yielding blocking artifacts at low bit rate. However, these methods produce satisfying results under an unreasonable assumption that the noise magnitude has been given. Several methods propose a block sensitive index to produce objective quality judgment according to observation. These methods require an original image as a reference and because of this requirement, they are too harsh too be used. A novel lossless compression model must be introduced to precisely measure the visibility of blocking artifacts and thus could be used to adjust the filter parameters for blocking artifacts reduction.

\section{Lossless Compression Technique}

Medical images are mostly used in radiological applications that are analyzed by a radiologist. Thus, all image compression approaches make assure that there is no loss of diagnostic information (datasets) for analysis and visual examination. The proposed method aims to remove blocking artifacts from medical images which are degraded by quantization noise by examining the characteristic of every region. The proposed method suggests one-dimensional filtering across the boundaries of $8 \times 8$ block. The filtering technique consists of various functional blocks, i.e. threshold value, low frequency model, moderate frequency model and high frequency model. Fig.1 shows the pixel vectors (PV) measured horizontally and vertically block boundaries. The vertical blocking artifacts are removed using the same procedure as the horizontal one. When blocking artifacts are processed vertical, just require to rotate an image by 90 degree and process the same as horizontal deblocking technique.

\section{A. Blocking Artifacts Measurement in Medical images}

To avoid any over- smoothing, blurs and to retain sharp edges within the block boundary a good quality of lossless compression algorithm should be adopted. In order to achieve this, threshold value should be constant. Although, due to degradation effect all discontinuities at block boundary are because a strong relationship exists between DC quantization parameter (QP) and threshold. The threshold value is given below: -

$$
\mid \text { offset } \mid \leq \mathrm{T}_{\mathrm{k}}
$$

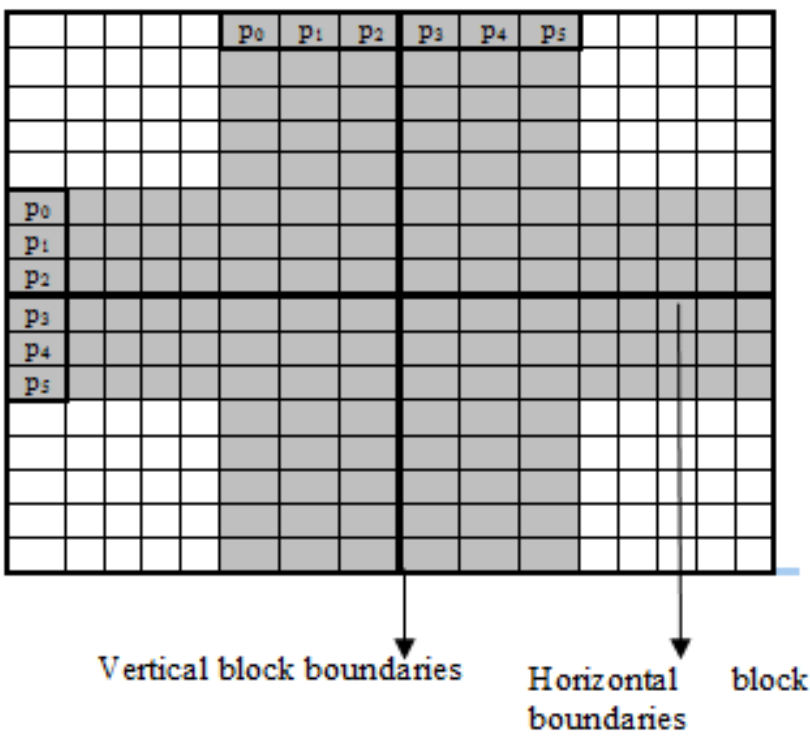

Fig 1: Pixel vectors for calculating activity

Where $\mathrm{T}_{\mathrm{k}}=155 \times \mathrm{QP}^{(0.09)}$, offset $=\left|\mathrm{p}_{2}-\mathrm{p}_{3}\right|$

Where $p_{2}$ and $p_{3}$ are pixel vector across the block boundary region as illustrated in fig $1 . T_{k}$ is used as a threshold to determine $8 \times 8$ adjacent blocks with blocking effects, $\mathrm{k}$ notation extract from the $\mathrm{k}^{\text {th }}$ pixel vector. $T_{k}$ should not be dependent only on QP, but it also depends upon local values. The strength of blocking effect nearby 
the block boundary is evaluated by analyzing the pixel among two horizontally adjacent blocks or vertically adjacent blocks. It is known as block boundary activity (BBA) and it is donated by $\forall(\mathfrak{b})$ is measured as:

$$
\begin{aligned}
& \forall(\mathfrak{v})=\sum_{K=0}^{4}\left[\varphi\left(p_{k}(i)\right]\right. \\
& \varphi\left(p_{k}(i)\right)=\left\{\begin{array}{l}
0, p_{k}(i) \in \alpha \\
1, \text { otherwise }
\end{array}\right.
\end{aligned}
$$

Where $\varphi($.$) is the indicator function, \alpha$ is a set,

$$
\alpha=\left\{p_{k}(\mathrm{i}):\left|p_{k}(i+1)-p_{k}(i)\right| \leq\right.
$$
$\Psi\}, \Psi$ is a threshold value which determines activity of neighboring pixels. After measuring, the five difference values with respect to Eq. (2) their sum is used to find an appropriate activity. For that the activity across the block boundary is a measure which is given in Eq. (2). $\alpha$ is inactivity constraint which is used to determine the relationship among two adjacent pixels. If activity across the block boundary is low, this determines a low frequency region while the high activity determines a high frequency region. If the activity lies between low and high activity, the region is called moderate frequency region.

\section{1) Low frequency model}

If the two $(8 \times 8)$ adjacent blocks have same frequency features and does not have high frequency components then it is considered as low frequency region. If $\forall(\mathfrak{p})$ is smaller than $T_{1}$ ( $T_{1}$ is threshold value to compare with $\forall(\mathfrak{b})$ for low frequency model) than pixel vector belongs to low frequency region. Low frequency model filter has less complexity as compared to moderate and high frequency model. If the region has low frequency:

$$
\text { If } \mid \text { offset } \mid<T_{L F M}
$$

Where $T_{L F M}$ is threshold value for the low frequency model. Here, designed another threshold value $\left(T_{H V}\right)$ to extract the pixel vectors with artifacts at a block boundary with constraint determined as:-

if $\mid$ off set $\mid \geq T_{H V}$

If the above constraint is satisfied, the pixel, vector is modified as Eq. (6). On the other hand, if above constraint is not satisfied, the pixel vector is modified as Eq. (7). For simplicity, $T_{H V}$ is set to $0.015 T_{k}$.

$$
\begin{aligned}
& p_{k}^{\prime}(\mathrm{i})=p_{k}(\mathrm{i})+\frac{\text { offset }}{\alpha_{i}}, i=0,1 \ldots . . N-3 \\
& \text { where } \alpha_{i}=\{8,6,2,-2,-6,-8\} \\
& p^{\prime}{ }_{k}(\mathrm{i})=p_{k}(\mathrm{i})+\frac{\text { offset }}{\alpha_{i}}, i=0,1 \ldots . . N-3
\end{aligned}
$$

The reduction of blocking artifacts is carried out by modifying six DCT coefficients (three on either side of the block boundary). On the other hand, if the condition given in Eq (4) is not satisfied, a new filter with another threshold value to identify two types of low frequency model is added to further nature improve the visual quality as well as preserve the image detail. The proposed edge filter with a threshold value $T_{L F M}$ where $T_{L F M}=0.11 T_{k}$ is as given in Eq. (8).

$$
P_{2}=p_{2}+\frac{2 \times o f f s e t}{1+\text { sqrt }(8 \times o f f s e t)}, P_{3}=p_{3}-\frac{2 \times o f f s e t}{1+\text { sqrt }(8 \times \text { offset })}
$$

Where $p_{2}, p_{3}$ are pixel vectors as illustrated in fig. 1 and $P_{2}, P_{3}$ are updated pixel vectors after implemented the filter near the block boundary.

\section{2) Moderate frequency model}

If the frequency of two $(8 \times 8)$ adjacent blocks exists between low frequency and high frequency, the region is considered as moderate frequency region. If $T_{1}<\forall(v)<T_{2} .\left(T_{1}\right.$ as earlier defined and $T_{2}$ is another threshold value) satisfied, then the region is determined as moderate frequency region. After calculating activity $F(V)$, two thresholds i.e. $T_{1}$ and $T_{2}$ are used to define the frequency mode. Experiments exhibit the settings as $T_{1}, T_{2}$. $\Psi$ to $1,3,2$ respectively. Moderate frequency region has higher activity than low frequency region. That is why it has a higher probability of using as given above in Eq. (8) an edge filter. If $\mid$ offset $\mid<T_{M F M}$, the region is moderate frequency model and filter defined by equation's (9) or (10) is activated, otherwise filter defined in Eq. (8) is activated. The edge filter on moderate frequency mode is also used. 


$$
\begin{gathered}
P_{1}=\frac{p_{1}}{4}+p_{0}+p_{1}+p_{2}, \quad P_{2}=\frac{p_{2}}{4}+p_{1}+p_{2}+p_{3}, \text { (9) } \\
P_{3}=\frac{p_{3}}{4}+p_{2}+p_{3}+p_{4}, \quad P_{4}=\frac{p_{4}}{4}+p_{3}+p_{4}+p_{5} \\
P_{2}=\frac{\text { offset }}{-10}+p_{2}, \quad P_{3}=\frac{\text { offset }}{10}+p_{3}
\end{gathered}
$$

Where $p_{1}, p_{2}, p_{3}, p_{4}$ are pixel vectors as illustrated in fig. 1 and $P_{1}, P_{2}, P_{3}, P_{4}$ are modified pixel vectors after implemented the filter in Eq.(9) and (10).$T_{M F M}$ is kept higher than $T_{L F M}\left(T_{M F M}=0.9 T_{k .}\right)$.

\section{3) High frequency model}

If frequencies of two $(8 \times 8)$ adjacent blocks differ from each other, the region is known as high frequency model. If $\forall(\mathfrak{y})>T_{2}$. is satisfied, then the region is considered as high frequency region. To remove over smoothing, across the block boundary, three thresholds $T_{H V}$ (As earlier defined, $T_{H F M}$, and $T_{d}$ are proposed. $T_{H F M}$ threshold for high frequency region and as used to analyse the edge filter by accepting edge threshold value and to preserve the true edges. If the difference between two adjacent pixels within block boundary is smaller than $T_{d}$ then the adjacent pixel values are updated according to Eq.'s (12) and (13). If $\mid$ off set $\mid<T_{H F M}$, the region is non smooth mode and the filter applies as defined in equation sets (8) and (11) with respect to the Eq. (4) are activated. Otherwise the filter is used as given in Equation's (12) and (13) are activated. Hence, analysis the difference between adjacent blocks and design a filter: -

$$
\begin{aligned}
& P_{2}=p_{2}-\frac{2 \times \text { offset }}{1+\text { sqrt }(8 \times \text { offset })},(11) \\
& \qquad P_{3}=p_{3}+\frac{2 \times \text { offset }}{1+\text { sqrt }(8 \times \text { offset })} \\
& P_{2}=\left\{\begin{array}{l}
p_{2}-\frac{\text { offset }}{8}, \text { if }\left(p_{2}-p_{3}\right) \leq T_{d} \\
p_{2}+\frac{\text { offset }}{8}, \text { otherwise }
\end{array}\right. \\
& P_{3}=\left\{\begin{array}{l}
p_{3}+\frac{\text { offset }}{4}, \text { if }\left(p_{3}-p_{4}\right) \leq T_{d} \\
p_{3}-\frac{\text { offset }}{4}, \text { otherwise }
\end{array}\right.
\end{aligned}
$$

Where $p_{2}, p_{3}, p_{4}$ are pixel vectors as illustrated in fig. 1 and $P_{2}, P_{3}$ are modified pixel vectors after implemented the above defined filters. Parameters $T_{H F M}$ and $T_{d}$ are defined empirically and determined as follows: $T_{H F M}=$ $0.7 T_{k}$ and $T_{d}=0.6 T_{k}$ respectively.

\section{B) Directional Filter}

Mostly edge blocks are affected by ringing artifacts and corner outliers, which are very difficult to eliminate by using one dimensional method. Thus, an appropriate method is to design a two-dimensional (2D) filter which is able to remove such types of artifacts and maintain the image quality. A $3 \times 3$ directional filter is used to filter the pixels $p_{2}$ and $p_{3}$ on either side of block boundary. In $3 \times 3$ mask filtering window only those pixels are modified which lies at block boundaries and a possible real edge must be preserved when the blocking artifacts is filtered. A 2D directional filtering with respect to $3 \times 3$ mask is as shown in fig. 2 . Suppose $f(\beta, \gamma)$ is the pixel to be filtered. Then the filtered output $f^{\prime}\left(\beta^{\prime}, \gamma^{\prime}\right)$ is obtained by using Eq. (14).

$$
f^{\prime}\left(\beta^{\prime}, \gamma^{\prime}\right)=\sum_{(\beta, \gamma) \in W\left(\beta^{\prime}, \gamma^{\prime}\right)} \mathfrak{w}(\beta, \gamma) f\left(\beta^{\prime}+\beta, \gamma^{\prime}+\gamma\right)
$$

Where $\mathfrak{w}(\beta, \gamma)$ is the weight function which is obtained by using the function of differences between the pixels as defined in Eq. (15).

\begin{tabular}{|l|l|l|}
\hline$(\beta-1, \gamma-1)$ & $(\beta-1, \gamma)$ & $(\beta-1, \gamma+1)$ \\
\hline$(\beta, \gamma-1)$ & $(\beta, \gamma)$ & $(\beta, \gamma+1)$ \\
\hline$(\beta+1, \gamma-1)$ & $(\beta+1, \gamma)$ & $(\beta+1, \gamma+1)$ \\
\hline
\end{tabular}

Fig. 2. A $3 \times 3$ mask for the $2 \mathrm{D}$ directional filtering for edge block

$$
\begin{array}{r}
\mathfrak{w}(\beta, \gamma)= \\
\exp \left(-\left|f\left(\beta^{\prime}+\beta, \gamma^{\prime}+\gamma\right)-f\left(\beta^{\prime}-\beta, \gamma^{\prime}-\gamma\right)\right|\right) / 2 \quad(15)
\end{array}
$$




\section{Experimental Results}

To illustrate the performance of the proposed method, it has been applied to JPEG compressed $(512 \times 512)$ medical images where original images are shown in fig.3. The experiment is conducted on four medical images namely CT, MRI, US and X-rays. Yimet. al.[16] proposed referenced quality assessment method named Peak Signal to Noise Ratio including blocking effects (PSNR-B). PSNR-B introduced a blocking effect factor

TABLE 1Psnr-B Values In Comparison With Different Deblocking Techniques.

\begin{tabular}{lllllllll}
\hline Image & Bit rate & JPEG & Proposed & $\begin{array}{l}\text { Chen et. } \\
\text { al. }[12]\end{array}$ & $\begin{array}{l}\text { Luo and } \\
\text { Ward [13] }\end{array}$ & $\begin{array}{l}\text { Singh } \text { et. al. } \\
{[14]}\end{array}$ & $\begin{array}{l}\text { Wang et. } \\
\text { al. }[15]\end{array}$ \\
\hline \multirow{3}{*}{ CT } & 0.275 & 27.85 & 29.56 & 25.92 & 27.86 & 27.80 & 28.39 \\
& 0.208 & 26.63 & 28.31 & 24.48 & 26.65 & 26.63 & 27.67 \\
\multirow{4}{*}{ MRI } & 0.169 & 25.79 & 27.21 & 23.19 & 25.82 & 25.89 & 26.61 \\
& 0.221 & 30.09 & 32.63 & 31.23 & 30.12 & 31.09 & 31.80 \\
& 0.139 & 26.42 & 28.70 & 27.26 & 26.49 & 27.21 & 28.63 \\
\multirow{4}{*}{ US } & 0.105 & 24.54 & 26.63 & 24.75 & 24.64 & 24.73 & 26.26 \\
& 0.151 & 25.35 & 27.49 & 26.25 & 25.40 & 26.22 & 27.30 \\
& 0.131 & 24.16 & 26.20 & 24.95 & 24.21 & 24.97 & 26.10 \\
\multirow{3}{*}{ X-ray } & 0.114 & 23.61 & 25.49 & 24.38 & 23.66 & 24.41 & 25.17 \\
& 0.122 & 27.75 & 29.24 & 28.68 & 27.76 & 28.61 & 29.07 \\
& 0.112 & 27.37 & 28.92 & 28.32 & 27.38 & 28.25 & 28.28 \\
& 0.104 & 27.10 & 28.68 & 28.02 & 27.12 & 28.00 & 28.06 \\
\hline
\end{tabular}

TABLE IIMssim Values In Comparison With Different Deblocking Techniques.

\begin{tabular}{|c|c|c|c|c|c|c|c|c|}
\hline Image & Bit rate & JPEG & Proposed & $\begin{array}{l}\text { Chen et. } \\
\text { al.[12] }\end{array}$ & $\begin{array}{l}\text { Luo and } \\
\text { Ward[13] }\end{array}$ & $\begin{array}{l}\text { Singh } \\
\text { al. }[14]\end{array}$ & et. & $\begin{array}{l}\text { Wang et. } \\
\text { al. }[15]\end{array}$ \\
\hline & 0.275 & 0.8808 & 0.8878 & 0.8863 & 0.8799 & 0.8809 & & 0.8648 \\
\hline \multirow[t]{3}{*}{$\mathrm{CT}$} & 0.208 & 0.6812 & 0.6904 & 0.6867 & 0.6803 & 0.6822 & & 0.6738 \\
\hline & 0.169 & 0.6495 & 0.6596 & 0.6550 & 0.6487 & 0.6501 & & 0.6460 \\
\hline & 0.221 & 0.8720 & 0.8971 & 0.8906 & 0.8718 & 0.8861 & & 0.8965 \\
\hline \multirow[t]{3}{*}{ MRI } & 0.139 & 0.7512 & 0.7973 & 0.7766 & 0.7520 & 0.7726 & & 0.7970 \\
\hline & 0.105 & 0.7066 & 0.7450 & 0.7117 & 0.7073 & 0.7082 & & 0.7392 \\
\hline & 0.151 & 0.7197 & 0.7518 & 0.7348 & 0.7197 & 0.7308 & & 0.7518 \\
\hline \multirow[t]{3}{*}{ US } & 0.131 & 0.6725 & 0.7090 & 0.6898 & 0.6726 & 0.6860 & & 0.7076 \\
\hline & 0.114 & 0.6219 & 0.6607 & 0.6405 & 0.6222 & 0.6376 & & 0.6552 \\
\hline & 0.122 & 0.5888 & 0.6206 & 0.6079 & 0.5893 & 0.6048 & & 0.6205 \\
\hline \multirow[t]{2}{*}{ X-ray } & 0.112 & 0.5786 & 0.6198 & 0.5974 & 0.5789 & 0.5952 & & 0.6170 \\
\hline & 0.104 & 0.5869 & 0.6177 & 0.6044 & 0.5871 & 0.6037 & & 0.6152 \\
\hline
\end{tabular}

TABLE IIIMOS Values In Comparison With Different Deblocking Techniques.

\begin{tabular}{lllllllll}
\hline Image & Bit rate & JPEG & Proposed & $\begin{array}{l}\text { Chen et. } \\
\text { al. [12] }\end{array}$ & $\begin{array}{l}\text { Luo and } \\
\text { Ward[13] }\end{array}$ & $\begin{array}{l}\text { Singh } \text { et. al. } \\
{[14]}\end{array}$ & $\begin{array}{l}\text { Wang et. } \\
\text { al. [15] }\end{array}$ \\
\hline \multirow{3}{*}{ CT } & 0.275 & 1.50 & 2.87 & 2.62 & 1.62 & 2.75 & 2.50 \\
& 0.208 & 1.37 & 2.62 & 2.37 & 1.50 & 2.50 & 2.25 \\
\multirow{4}{*}{ MRI } & 0.169 & 1.25 & 2.37 & 1.75 & 1.25 & 2.12 & 2.00 \\
& 0.221 & 1.62 & 3.62 & 3.37 & 1.87 & 3.12 & 3.00 \\
& 0.139 & 1.25 & 3.25 & 2.87 & 1.62 & 2.75 & 2.12 \\
US & 0.105 & 1.00 & 2.75 & 2.50 & 1.00 & 1.87 & 2.00 \\
& 0.151 & 1.87 & 3.87 & 2.50 & 1.75 & 2.00 & 2.37 \\
& 0.131 & 1.62 & 3.37 & 2.25 & 1.37 & 1.75 & 2.00 \\
\multirow{4}{*}{ X-ray } & 0.114 & 1.00 & 2.87 & 2.00 & 1.00 & 2.50 & 2.12 \\
& 0.122 & 2.00 & 3.25 & 2.62 & 2.12 & 2.75 & 2.50 \\
& 0.112 & 1.75 & 3.00 & 2.12 & 1.87 & 2.00 & 1.62 \\
\hline
\end{tabular}




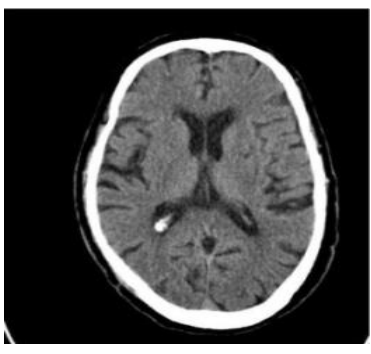

a)

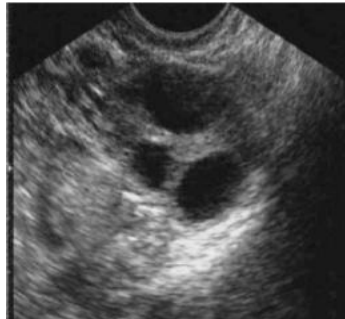

c)

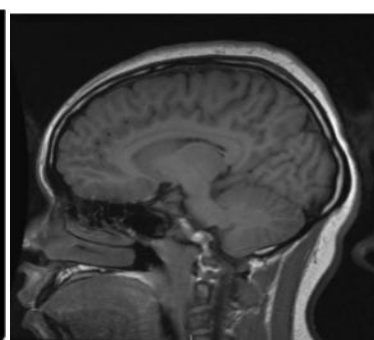

b)

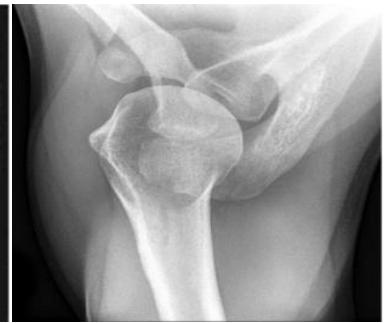

d)

Fig. 3. Test images.

(BEF) to measure the blocking effects of the test images, which is more effective for assessing the quality of deblocking images than Peak Signal to Noise ratio (PSNR). To evaluate the efficiency of the proposed technique, four existing methods; Chen et. al.[12], Luo and Ward[13], Singh et. al.[14] and Wang et. al.[15] have been implemented and the results are compared with the results of the proposed method. The methods demonstrated in Ref.[12-13] have presented similar results and mostly PSNR-B values have identical to decoded image. The proposed method gives better performance of $3.64 \mathrm{~dB}$ higher PSNR-B than the method proposed by Chen et. al.[12] and minimum improvement of $1.17 \mathrm{~dB}$ higher than Wang et. al.[15] at 0.275 bit-rate in case of CT image. The comparison of PSNR-B values for MRI image, the proposed technique gives the best performance of $2.51 \mathrm{~dB}$ higher than the method proposed by Luo and Ward [12] and lowest improvement of $0.83 \mathrm{~dB}$ than the method proposed by Wang et. al. [15] at 0.221 bit-rate. Similarly, the comparison of PSNR-B values for US images, the proposed method gives the best performance of $1.99 \mathrm{~dB}$ higher than the method proposed by Luo and Ward [13] and lowest improvement of $0.10 \mathrm{~dB}$ than the method proposed by Wang et. al. [15] at 0.131 bit-rate. For X-ray image, there is an improved of $0.63 \mathrm{~dB}$ on average case than the method proposed by Singh et. al. [14] at 0.122 bit-rate. Table I demonstrates the proposed method scheme exhibits the best PSNR-B values as compared to existing methods [12-15] for test images.

Wang et. al. [17] deigned an objective method which is based on quality measures from perspective of image formation. A mean SSIM (MSSIM) index is used to evaluate the overall image quality. The higher values of MSSIM index is determined the better quality of an image. From Table II demonstrates the MSSIM index values for test images. There is significant improvement in MSSIM index values for different medical images with respect to other compression methods. The method proposed by Luo and Ward [13] is observed that the reduction in MSSIM values. Therefore, this method indicates that it doesn't maintain the structural similarity of an image. Table II shows that the proposed scheme has largest MSSIM index values with respect to other conventional methods. The proposed method illustrated excellent performance as compared to conventional methods.

Mean Opinion Scores (MOS) [18] values collected from an experiment involving nine radiologists for distorted images. The original image without compression used as reference condition. The radiologist found that the images compressed with proposed method are better than other methods. Table III demonstrates the MOS values of the proposed method is also superior to other methods.

\section{Conclusion}

Medical image compression is unavoidable due to large amount of storage space or high bandwidth for communication in its original form. Medical imaging technique has become very important aid in medical practice because it allowing detailed observation of anatomical as well as physiological structures of the human body. Nevertheless, in medical applications the need to conserve the diagnostic validity of the image requires the use of lossless compression methods. If lossless compression technique is used for medical images, the compression ratio achieved is low. The lossy compression method restored the medical image may be lose datasets required for detection. A novel lossless compression technique, which removes blocking effects from an image while maintaining all relevant structure, has been proposed. The advantage of the proposed method with respect to other 
compression methods, it compress-decompress images while removing the blocking artifacts. Experimental results demonstrate that the proposed compression lossless technique generates good fine detail of an image and has improved the compression ratio.

\section{References}

Z. Zuo, X. Lan, L. Deng, S. Yao, and X. Wang, “An improved medical image compression technique with lossless region of interest," Optik, vol. 126, pp. $2825-2831,2015$.

B. K. Chandrika, P. Aparna, and David S. Sumam, "Perceptually lossless coder for volumetric image data, " J. Vis commun. Image R.,vol. 46, pp. 23-32, 2017.

Binh P. Nguyen, Chee-Kong Chui, Sim-Heng Ong, and Stephen Chang, "An efficient compression scheme for 4D medical images using hierarchical vector quantization and motion compression, " Computers in Biology and Medicine, vo. 41, pp. 843-856, 2011.

Nan Jiang, and Yi Zhuang, Dickson K.W. Chiu, "Multiple transmission optimization of medical images in recourse-constraint mobile telemedicine systems," Computer Methods and Programs in Biomedicine, vol. 145, pp. 103-113, 2017.

Vikash Yadav, Monika Verma, and Vandana Dixit Kaushik, "A hybrid image compression technique for medical images," IEEE Conference on Computational Intelligence and Communication Network, 2015, pp. 222-227.

Luis F. R. Lucas, Nuno M. M. Rodrigues, Luis A. da Silva Cruz, and Sergio M. M. de Faria, "Lossless compression of medical images using 3D predictors," IEEE Transactions on Medical Imaging, vol. pp. 1-11, 2017.

G. Placidi, "Adaptive compression algorithm from projections: Application on medical greyscale images," Computers in Biology and Medicine, vol. 39, pp. 993-999, 2009.

R. Sumalatha, and M. V. Subramanyam, "Hierarchical lossless image compression for telemedicine applications," Procedia Computer Science, vol. 54, pp. 838-848, 2005.

Sukhwinder Singh, Vinod Kumar, and H. K. Verma, "Adaptive threshold-based block classification in medical image compression for teleradiology," Computers in Biology and Medicine, vol. 37, pp. 811-819, 2007.

D. Ravichandran, Ramesh Nimmatoori, and AshwinDhivakar M R, "Performance of wavelet based image compression on medical images for cloud computing," IEEE International Conference on Computing for Sustainable Global Development, 2016, pp. 297-302.

Jagroop Singh, Sukhwinder Singh, Dilbag Singh, and Moin Uddin, "Detection method and filters for blocking effect reduction of highly compressed images," Signal Processing: Image Communication, vol. 26, no.8-9, pp. 493-506, 2011.

Yen-Yu Chen, Ying-Wen Chang, and Wen-Chien Yen, "Design a deblocking filter with three separate modes in DCT-based coding," Journal of Visual Communication and Image Representation, vol. 19, pp. 231-244, 2008

Ying Luo, and K. Rabab Ward, "Removing the blocking artifacts of block-based DCT compressed images," IEEE Transactions on Image Processing, 2003, pp. 838-842.

Jagroop Singh, Sukwinder Singh, Dilbag Singh, and Moin Uddin, "A signal adaptive filter for blocking effect reduction of JPEG compressed images," International Journal of Electronic and Communications, vol. 65, pp. 827-839, 2011.

Jin Wang, Wu Zhensen, GwanggilJeon, and JechangJeong, "An efficient spatial deblocking of images with DCT compression,” Digital Signal Processing, vol. 42, pp. 80-88, 2015.

ChanghoonYim, and Alan Conrad Bovik, "AC Quality assessment of deblocked images," Transactions on Image Processing, vol. 20, no. 1, pp. 88-98, 2011.

Zhou Wang, Alan Conrad Bovik, Hamid R. Sheikh, and Eero P. Simoncelli, "Image quality assessment: from error visibility to structural similarity," Transactions on Image Processing, vol. 13, no. 4, pp. 1-14, 2004.

S. Grgic, and M. Grgic, "Reliability of objective picture quality measurement," Journal of Electrical Engineering, vol. 55, no.1-2, pp. 3-10, 2004. 